\title{
Modification of Antitumor Immunity by Supernatants of oncoytic Herpes Simplex Virus-Infected Squamous Cell Carcinoma Cells
}

\author{
Shinya Tada ${ }^{1}$, Masakazu Hamada ${ }^{1}$, Yoshiaki yura ${ }^{1 *}$ \\ ${ }^{1}$ Department of Oral and Maxillofacial Surgery, Osaka University Graduate School of Dentistry, Suita, Osaka, Japan
}

Received: 27Novmber, 2017; Accepted: 29 January , 2018; Published: 05 February, 2018

*Corresponding authors: Dr. Yoshiaki Yura, Department of Oral and Maxillofacial Surgery, Osaka University Graduate School of Dentistry, Suita, Osaka 5650871, Japan, Tel: +81-06-6879-2941; Fax: +81-06-6879-2170; E-mail: narayura630@gmail.com

\begin{abstract}
Aims: To determine whether the supernatants of oncolytic herpes simplex virus type 1 (HSV-1)-infected squamous cell carcinoma (SCC) cells can stimulate cytotoxic T cells through dendritic cell (DC) maturation, in vitro and in vivo experiments were performed.

Introduction: Oncolytic HSV-1 strain RH2 induced immunogenic cell death with the release and surface exposure of damage-associated molecular patterns (DAMPs) in SCC SCCVII cells. The ability of the supernatants of RH2-infected SCCVII cells to activate immune cells was examined.
\end{abstract}

Materials and methods: The supernatants of SCCVII cells infected with RH2 and cultured for $24 \mathrm{hrs}$ and mock-infected cells were concentrated to produce Med24 and MedC. Splenocytes were prepared from tumor-bearing mice. Tumor necrosis factor $\alpha$ (TNF- $\alpha$ ) and interferon $\gamma$ (IFN- $\gamma$ ) were measured by enzyme-linked immuno sorbent assay (ELISA).

Results: The production of IFN- $\alpha$ was increased by a co-culture of $\mathrm{CD} 4^{+} \mathrm{T}$ cells or $\mathrm{CD} 8^{+} \mathrm{T}$ cells with Med24-treated DC. In a co-culture with SCCVII cells, the splenocytes of mice treated with an intratumoral injection of Med24 produced more IFN- $\gamma$ and exhibited greater cytotoxicity than those of MedC-treated mice. The intratumoral injection of Med24 also increased tumor-infiltrating CD8 ${ }^{+} \mathrm{T}$ cells.

Conclusion: These results suggest that the supernatants of HSV-1 RH2-infected SCC cells include the molecules responsible for the activation of DCs and T cells and promote anti-tumor immunity against SCC.

Keywords: Oncolytic Virotherapy; Herpes Simplex Virus; Antitumor Immunity;

\section{Introduction}

Oncolytic virotherapy is a novel therapeutic modality that directly induces the lysis of infected tumor cells and subsequently enhances host immune responses [1,2,3,4,5]. Herpes simplex virus type 1 (HSV-1) is one of the most widely studied oncolytic viruses for the treatment of patients with solid tumors. [6,7].

The activation of dendritic cell (DC) may be directly induced by infectious agents and indirectly by inflammatory products. One of the main roles of DCs in the induction of adaptive immunity is antigen presentation. Captured antigens are processed by the endocytic pathway of DCs and loaded on major histocompatibility complex (MHC) molecules [8]. MHC class II-rich compartment in DCs allows for the rapid presentation of exogenous antigens for the generation of $\mathrm{CD} 4^{+} \mathrm{T}$ helper cells [9]. In the activation of cytotoxic CD8 ${ }^{+} \mathrm{T}$ cells, DCs present endogenous antigens via class I MHC molecules following direct infection, as observed during infections by HSV [10]. CD $4^{+} \mathrm{T}$ cells are required for the priming of CD8 ${ }^{+} \mathrm{T}$ cells after HSV-1 infection [11].

Previously, we reported that an injection of oncolytic HSV1 strain RH2 into squamous cell carcinoma cell (SCC) SCCVII tumors in inbred mice was shown to reduce the growth of injected and non-injected tumors and enhance the activity of $\mathrm{CD}^{+} \mathrm{T}$ cells [12]. We also indicated that the supernatants of RH2infected cells suppressed tumor growth, even if infectivity was lost by ultraviolet irradiation, suggesting the ability of molecules secreted from HSV-1-infected tumor cells to enhance antitumor immunity [13]. However, the role of DC in RH2-mediated enhancement of antitumor immunity remains unknown. In the present study, we examined the ability of the supernatants of oncolytic HSV-1 RH2-infected SCC cells to stimulate cytotoxic T cells through DC maturation.

\section{Materials and methods}

\section{Cells and virus}

SCCVII cells derived from the cutaneous SCC of C3H mice were cultured with Eagle's minimal essential medium (MEM) supplemented with $10 \%$ calf serum at $37^{\circ} \mathrm{C}$ in a humidified atmosphere with $5 \% \mathrm{CO}_{2}[12,13]$. Yac-1 lymphoid cells induced by Molony virus in A/Sn mice were obtained from the Riken Cell Bank (Ibaraki, Japan) and cultured in RPMI 1640 supplemented with $10 \%$ fetal bovine serum (FBS). HSV-1 strain RH2 lacking the $\gamma 34.5$ gene and with multiple mutations including glycoprotein $B$ were grown in Vero cell monolayers and infectivity was assessed using a plaque assay on a Vero cell monolayer [12,14].

\section{Preparation of concentrated supernatants of RH2- infected cells}

SCCVII cells were infected with RH2 at a multiplicity of infection (MOI) of 10. After being incubated for 60 min for virus 
adsorption, cell monolayers were washed twice with phosphatebuffered saline (PBS), covered with serum-free medium, and cultured for $24 \mathrm{~h}$. Culture supernatants were then harvested, and centrifuged at 1,500 rpm for $10 \mathrm{~min}$ to remove cell debris. The supernatants were concentrated 30 times using Amicon $® U l t r a-15$ 3K Centrifugal Filter Devices (Merck) [13]. Concentrates were exposed to ultraviolet irradiation at an intensity of $0.15 \mathrm{~mW} / \mathrm{cm}^{2}$ for $30 \mathrm{~min}$ in order to inactivate the infectivity of the virus, filtered through a $0.20-\mu \mathrm{m}$ filter, and named Med24. Mock-infected cells were also incubated in serum-free medium, supernatants were harvested $24 \mathrm{~h}$ later, treated as described for RH2- infected cells, and named MedC.

\section{Tumor induction and tumor treatment with Med24 and MedC}

In order to prepare splenocytes from tumor-bearing mice, 5-week-old $\mathrm{C} 3 \mathrm{H} / \mathrm{HeJJcl}$ female mice were obtained from Clea Japan (Tokyo, Japan) and $1 \times 10^{6}$ SCCVII cells were injected subcutaneously into the backs of these mice. In experiments involving treatments with Med24 and MedC, tumors were generated in both legs by a subcutaneous injection of $1 \times 10^{6}$ SCCVII cells. After the longer axis of tumors reached $5 \mathrm{~mm}, 100 \mu \mathrm{l}$ of Med24 or MedC was administered to one side of the leg tumor three times at 3-day intervals. Contralateral leg tumors were left untreated. Fourteen days after the start of the treatment, the splencytes of mice were prepared. Experiments were conducted with the approval of the Institute of Laboratory.

\section{Preparation of DCs, CD4 ${ }^{+} \mathrm{T}$ cells, and $\mathrm{CD8}^{+} \mathrm{T}$ cells from tumor-bearing mouse spleens}

SCCVII tumors were produced on the backs of mice and spleens were removed after tumors reached approximately 5 $\mathrm{mm}$ in diameter. The tissue homogenate was passed through a mesh. Splenocytes were overlayed on Lympholyte $®-M$ (Cedarlane, Burlington, Canada), centrifuged at 3,500 rpm for $20 \mathrm{~min}$, and the leukocyte layer obtained was harvested. Splenocytes were then washed with PBS and suspended in serum-free RPMI 1640 medium. They were reacted with CD11c MicroBeads (MiltenyiBiotec) at $4{ }^{\circ} \mathrm{C}$ for $15 \mathrm{~min}$ and applied to an LS column (MiltenyiBiotec) to recover CD11c ${ }^{+}$DCs [15]. DCs were resuspended in RPMI 1640 medium supplemented with $10 \mathrm{ng} /$ $\mathrm{ml}$ granulocyte macrophage colony-stimulating factor, $10 \mathrm{ng} / \mathrm{ml}$ interleukin (IL)-4, and 10\%FBS [16]. In order to separate CD4+ $\mathrm{T}$ cells, splenocytes were suspended in MACS Buffer, mixed with CD4 (L3T4) MicroBeads, and incubated at $4^{\circ} \mathrm{C}$ for $15 \mathrm{~min}$. After washing, CD $4^{+} \mathrm{T}$ cells were collected on the LS column by positive selection. Similarly, CD8 ${ }^{+}$T cells were isolated using CD8a (Ly-2) MicroBeads [17,18].

Treatment of DCs with Med24 and MedC and measurement oftumor necrosis factor $\alpha$ (TNF- $\alpha$ ) by an enzyme-linked immunosorbent assay (ELISA)

Spleen-derived DCs from tumor-bearing mice were plated at $2 \times 10^{5}$ /well on 24-well plates, mixed with Med24, MedC, or MEM in $500 \mu \mathrm{l}$ medium, and cultured for $48 \mathrm{~h}$. Samples from the supernatants of SCCVII cells were diluted 1:5 at this stage.
Alternatively, spleen-derived DCs from tumor-bearing mice were incubated with Med24 or MedC for $3 \mathrm{~h}$, with a sample dilution of 1:1.6. After the incubation, the culture medium of DCs was harvested and centrifuged at $1,500 \mathrm{rpm}$ for $10 \mathrm{~min}$ to remove cell debris and the amount of TNF- $\alpha$ in the culture medium was measured using Quantikine ${ }^{\circledR}$ ELISA mouse TNF- $\alpha$ (R \& D Systems) according to the manufacturer's instruction. The amount of TNF- $\alpha$ was measured at an absorbance of $450 \mathrm{~nm}$ with a microplate reader (Benchmark Plus, Bio-Rad).

\section{Co-culture of DCs and T cells and measurement of interferon $\gamma($ IFN- $\gamma)$ by ELISA}

Spleen-derived DCs from tumor-bearing mice were incubated with Med24 or MedC for $3 \mathrm{~h}$ and named Med24-DCs and MedCDCs. As a control, spleen-derived DCs were incubated in the presence of MEM, instead of Med24 and MedC, for $3 \mathrm{~h}$ and named MEM-DC. CD4 ${ }^{+} \mathrm{T}$ cells and $\mathrm{CD}^{+} \mathrm{T}$ cells from tumor-bearing mice were plated at $2 \times 10^{5} /$ well on 24 -well plates, mixed with pulsetreated DCs at a ratio of 9:1, and cultured for $48 \mathrm{~h}$. The amounts of IFN- $\gamma$ produced in the culture medium were measured using Quantikine ${ }^{\circledR}$ ELISA mouse IFN- $\gamma$. (R \& D Systems) according to the manufacturer's instructions.

In a co-culture study with T cells and cancer cells, mice with leg tumors were treated with intratumoral injections of Med24 or MedC on one leg, and splenocytes were prepared. SCCVII and Yac1 had been plated on 12-well plates $24 \mathrm{~h}$ previously. Thereafter, $2 \times 10^{6}$ splenocytes were mixed with tumor cells at a ratio of 20:1, and incubated at $37^{\circ} \mathrm{C}$ for $48 \mathrm{~h}$. IFN $-\gamma$ produced in the culture medium was measured using Quantikine $®$ ELISA mouse IFN- $\gamma$.

\section{Lactate dehydrogenase (LDH) release assay}

SCCVII cells and Yac- 1 cells had been plated at $5 \times 10^{3} /$ well on 96-well plates $24 \mathrm{~h}$ previously. Splenocytes were prepared from Med24-treated or MedC-treated mice, mixed with tumor cells at effector / target ratios of 5:1, 20:1, and 50:1, and cultured for $24 \mathrm{~h}$. Thereafter, the culture medium was harvested and cytoplasmic enzyme LDH release was evaluated using MTXLDH (Kyokuto, Tokyo, Japan) according to the manufacturer's instructions. In positive control wells, TritonX-100 was used at a final concentration of $0.8 \%$ to assess maximal LDH release. Percent cytotoxicity was calculated as $100 \times$ (experimental LDH - spontaneous LDH) / (maximum LDH release - spontaneous LDH) $[12,13]$. In order to minimize the effects of effector cells, splenocytes were cultured in the absence of cancer cells and the spontaneous release of LDH from splenocytes was assessed. These values were subtracted from the data obtained by the coculture.

\section{Flow cytometry}

Tumor tissues were homogenized and dissociated. Tumorinfiltrating cells were collected through meshes and suspended in RPMI 1640 supplemented with $10 \mathrm{U} / \mathrm{ml} \mathrm{IL-2.} \mathrm{Cells} \mathrm{were}$ reacted with anti-mouse CD8a Fluorescein Isothiocyanate (FITC) (Affymetrix) or Rat IgG2a K Isotype Control FITC (MiltenyiBiotec) at $4^{\circ} \mathrm{C}$ for $30 \mathrm{~min}$ under protection from light. After washing twice, 
stained cells were analyzed using a FACS Calibur flow cytometer (Becton-Dickinson) and Cell Quest software.

\section{Statistical Analysis}

Statistical analyses of two groups were performed using the Student's t- test with Microsoft Excel (Microsoft, Redmond, WA). For multiple comparisons, a one-way Analysis of Variance (ANOVA) and Student's t-test were used. Results were expressed as the mean \pm SD. Differences were considered to be significant at $\mathrm{P}<0.05$.

\section{Results}

\section{Production of TNF- $\alpha$ from DCs by concentrated supernatants of RH2-infected cells}

Several agents have been used to differentiate immature DCs into mature antigen-presenting cells $[15,19]$. After maturation, DCs acquire the ability to produce cytokines such as TNF- $\alpha$, IL-12, and IFN $[16,20,21]$. For preparation of concentrated supernatants of RH2-infected cells, SCCVII cells were infected with RH2 at a MOI of 10 . After $24 \mathrm{~h}$, culture supernatants were concentrated, exposed to ultraviolet irradiation in order to inactivate the infectivity of the virus, and named Med24. Supernatants of mock-infected cells were treated in a similar manner and named MedC. In order to investigate the effects of Med24 on DCs, DCs were prepared from the spleens of SCCVII tumor-bearing mice, incubated with concentrated supernatants for $48 \mathrm{~h}$, and the production of TNF- $\alpha$ was then evaluated by an ELISA. The mean

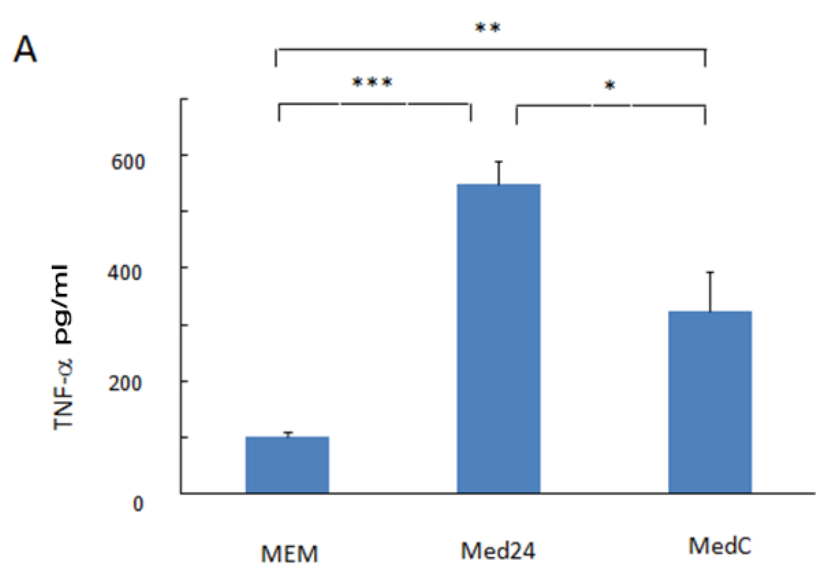

Figure 1A: Production of TNF- $\alpha$ from DCs by concentrated supernatants of RH2-infected cells and production of IFN- $\gamma$ by a co-culture of T cells and DCs. (A) DCs expressing CD11 were prepared from SCCVII tumorbearing mice and incubated with Med24 or MedC for $3 \mathrm{~h}$. The amounts of TNF- $\alpha$ produced in the culture medium were then measured. (B) DCs were pulse-treated with Med24 or MedC for $3 \mathrm{~h}$ to produce Med24-DC and MedC-DC. CD ${ }^{+}$T cells prepared from SCCVII tumor-bearing mice were mixed with Med24-DCs or MedC-DCs, and cultured for $48 \mathrm{~h}$. The amounts of TNF- $\alpha$ in the culture medium were then measured. (C) CD8 ${ }^{+}$ T cells wereco-cultured with Med24-DCs or MedC-DCs for $48 \mathrm{~h}$ and the amounts of IFN-in the culture medium were measured. The data are shown as the means \pm SD of values from triplicate cultures obtained in a single experiment and are representative of three independent experiments. ${ }^{*} \mathrm{P}<0.05,{ }^{* *} P<0.01,{ }^{* * *} P<0.01$. values of TNF- $\alpha$ in MEM alone, Med24, and MedC groups were $301 \mathrm{pg} / \mathrm{ml}, 434 \mathrm{pg} / \mathrm{ml}$, and $349 \mathrm{pg} / \mathrm{ml}$, respectively. The amount of TNF- $\alpha$ was increased by the incubation with Med 24, reaching $144 \%$ of the control (MEM alone). Even if DCs were pulse-treated with Med24 for $3 \mathrm{~h}$, a significant increase in TNF- $\alpha$ was noted (Figure 1A). Therefore, these pulse-treated DCs, Med24-DC and MedC-DC, were used in subsequent co-culture studies.

\section{Production of IFN- $\boldsymbol{\gamma}$ by a co-culture of T cells and DCs}

$\mathrm{CD}^{+} \mathrm{T}$ cells need to proliferate clonally and acquire function in order to eliminate virally infected or transformed cells $[22,23]$. In order to assess the effects of DCs on T cell activation, co-culture studies were conducted using Med24-DCs and MedC-DCs. Control DCs, named MEM-DCs, were incubated with MEM for 3 h. CD $4^{+} T$ cells and $\mathrm{CD}^{+} \mathrm{T}$ cells were also prepared from tumor-bearing mice. When CD4+T cells were co-cultured with Med24-DCs or MedC-DCs, IFN- $\alpha$ levels in the culture medium were increased as compared with control (MED-DC). The amounts of IFN- $\gamma$ in the culture medium of Med24-DCs and MedC-DCs groups were 135\% and $120 \%$ of the control (MEM-DC), respectively and a significant difference was observed between the Med24 and MedC groups (Figure 1B). When $\mathrm{CD}^{+} \mathrm{T}$ cells were co-cultured with DCs, more IFN- $\gamma$ was also produced in the Med24-DC group (Figure 1C). A significant difference was observed between the Med24-DC and MedC-DC groups.

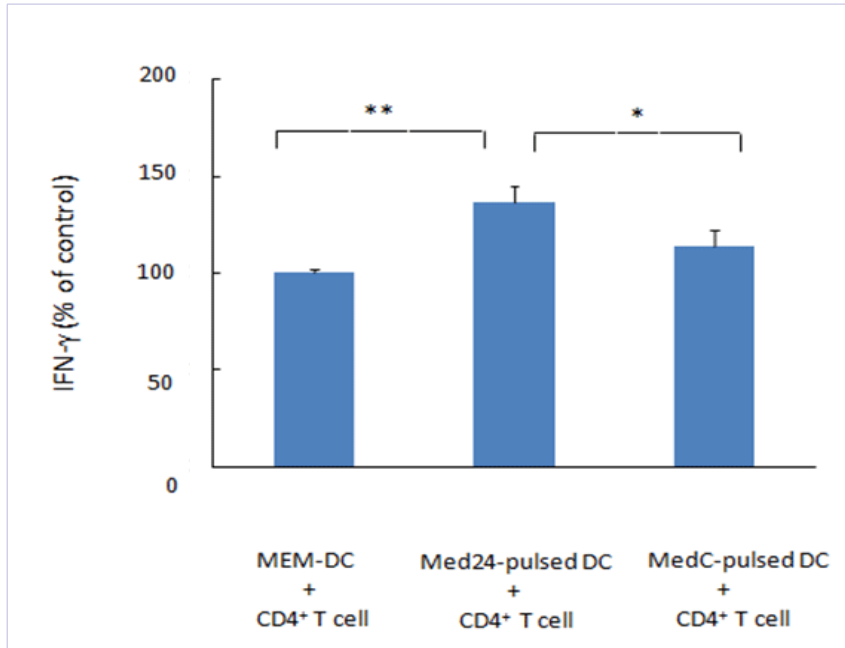

Figure 1B:

Effects of an intratumoral injection of Med24 on IFN- $\gamma$ producing ability and cytotoxic activity of splenocytes

Since DCs, CD4 ${ }^{+} \mathrm{T}$ cells, and $\mathrm{CD} 8^{+} \mathrm{T}$ cells were activated by Med24 in vitro, we investigated whether the antitumor ability of splenocytes may be activated by the in vivo administration of Med24. When SCCVII cells were co-cultured with the splenocytes of Med24-treated mice or MedC-treated mice, the amounts of IFN- $\gamma$ produced were $4,671 \mathrm{pg} / \mathrm{ml}$ and $1,554 \mathrm{pg} / \mathrm{ml}$, respectively. A 3-fold difference was noted between the Med24 and MedC groups (Figure2A). In contrast, the co-culture with Yac-1 cells did not increase the production of IFN $-\gamma$ from splenocytes. 

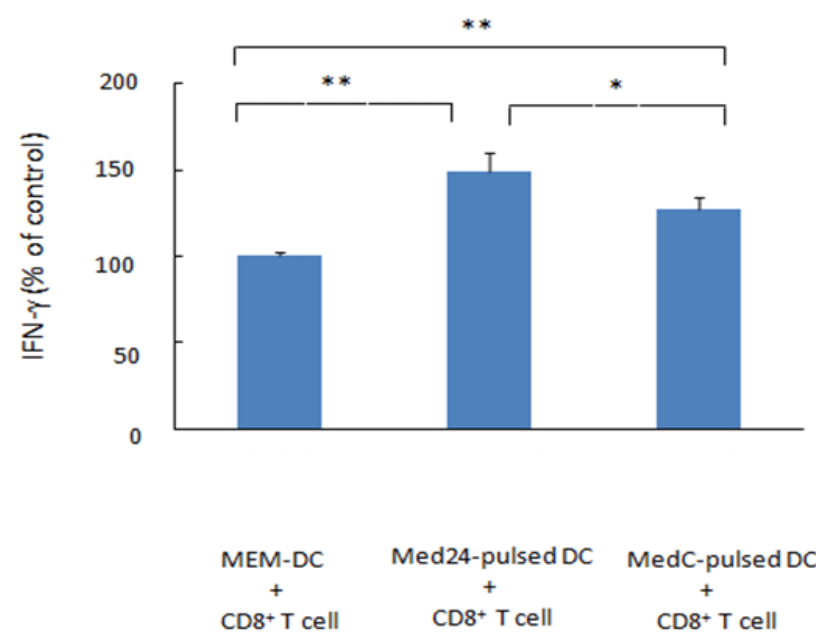

Figure 1C:

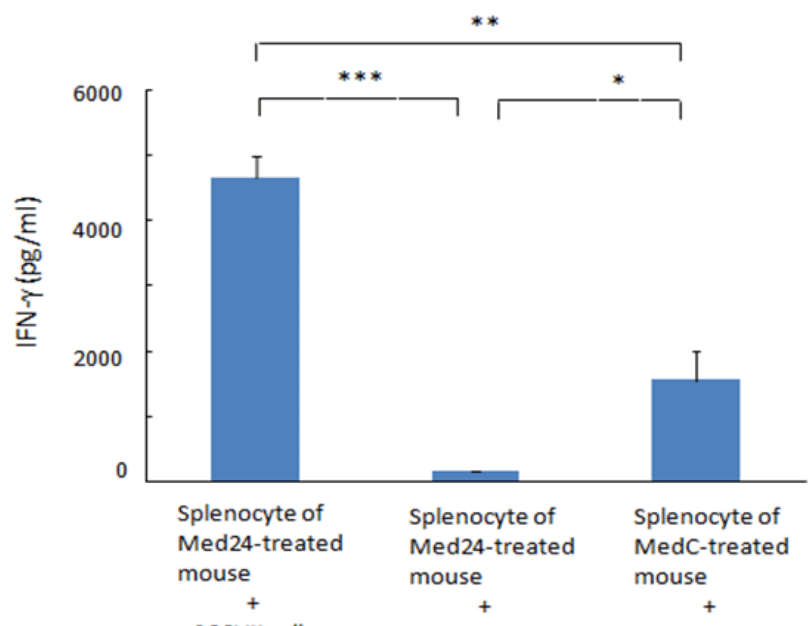

Figure 2A: Effects of an intratumor injection of Med24 on IFN- $\alpha$ producing ability and cytotoxic activity of splenocytes. (A) SCCVII tumors were produced subcutaneously on both legs. One side of tumor was treated three times with an intra tumoral injection of Med24 or MedC. Splenocytes were prepared 14 days after the start of the treatment, co-cultured with SCCVII cells or Yac- 1 cells for $48 \mathrm{~h}$, and the amounts of IFN- $\alpha$ in the culture medium were measured. (B) Splenocytes from Med24-treated or MedC-treated mice were mixed with SCCVII cells at a ratio of 5:1, 20:1, and 50:1 and cultured for $24 \mathrm{~h}$. The cytotoxicity of splenocytes was measured by an LDH release assay. The data are shown as the means \pm SD of values from triplicate cultures obtained in a single experiment and are representative of three independent experiments. ${ }^{*} P<0.05,{ }^{* *} P$ $<0.01,{ }^{* * *} P<0.01$.

LDH release assays were performed to assess the cytotoxicity of splenocytes to SCCVII cells. When splenocytes were cocultured with SCCVII cells at an effector / target ratio of 50:1, the values in the Med24 and MedC treatment groups were $25 \%$ and $6 \%$, respectively, indicating an increase in the cytotoxic ability of splenocytes in the Med24 group (Figure2B).
B

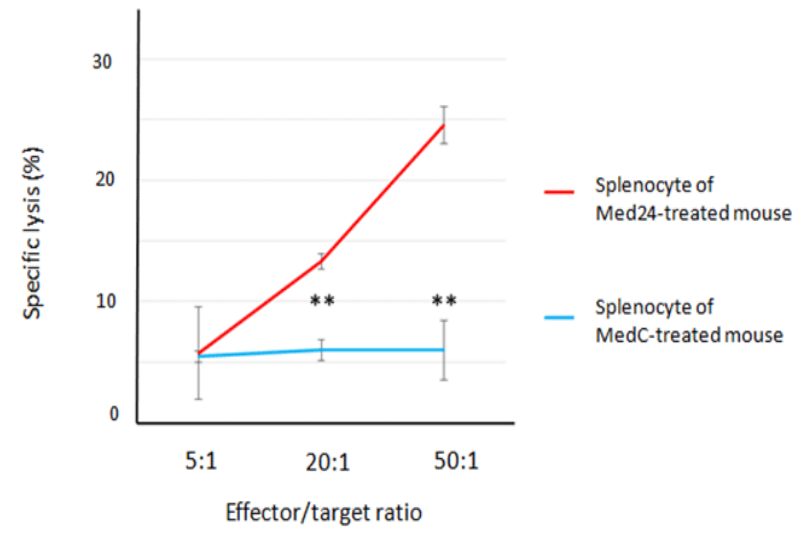

Figure 2B:

\section{Flow cytometry of $\mathrm{CD8}^{+} \mathrm{T}$ cells infiltrating tumors}

We then investigated whether an intratumoral injection of Med24 promoted CD8 ${ }^{+} \mathrm{T}$ cell infiltration into tumor tissues. Med24 or MedC were injected into one side of the leg tumor and $\mathrm{CD}^{+} \mathrm{T}$ cells infiltrating on both sides of the tumor were analyzed using flow cytometry (Figure 3A). On the injected side, the mean values of tumor-infiltrating $\mathrm{CD}^{+} \mathrm{T}$ cells in the Med24and MedC groups were $3.7 \%$ and $0.96 \%$, respectively. On the noninjected contralateral side, the mean values for the Med24 and MedC groups were $4.3 \%$ and $1.1 \%$, respectively (Figure3B). A significant difference was observed between the two groups on both sides of the tumor, indicating the systemic effect of Med24 on tumor immunity.

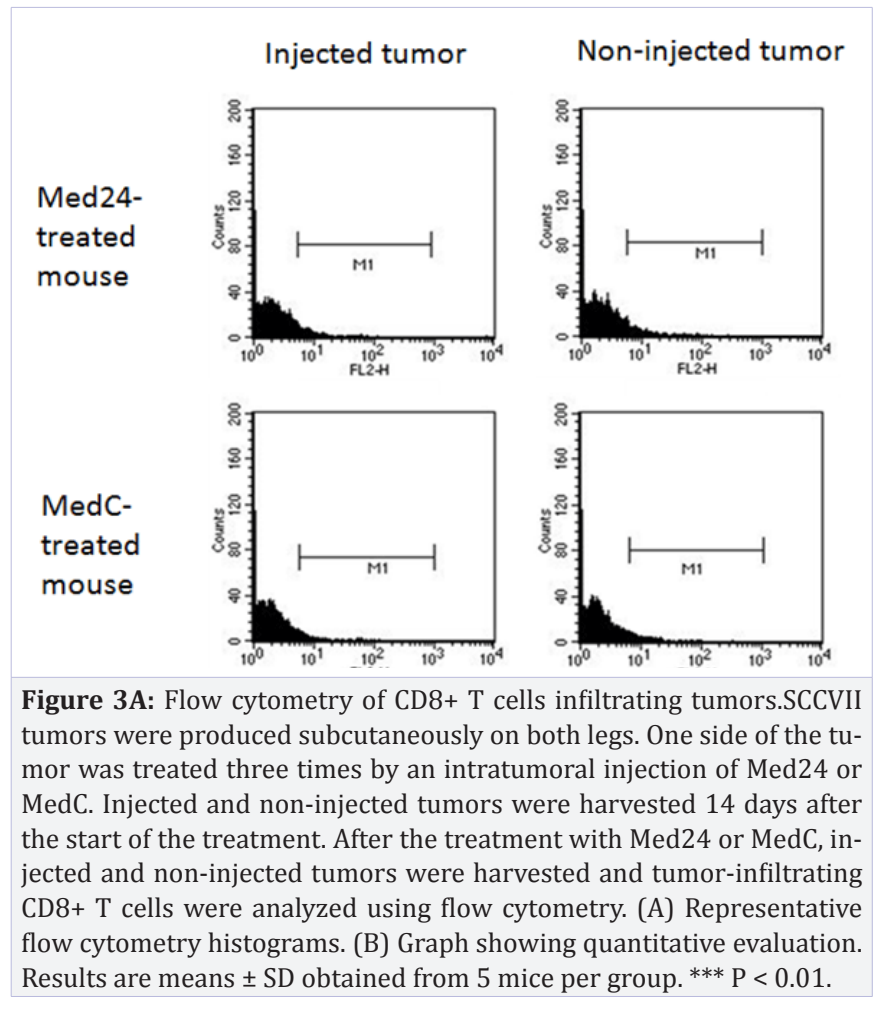




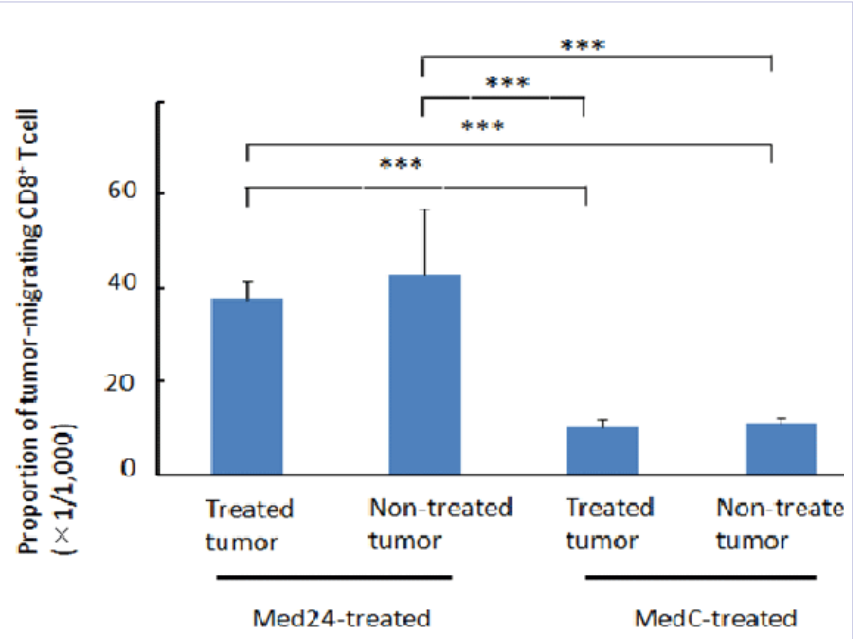

Figure 3B:

\section{Discussion}

Numerous oncolytic viruses are potent inducers of class I MHC pathway-related molecules, thus immediately providing possible tumor/viral immune recognition, providing possible tumor and viral immune recognition [24]. With respect to DCs, oncolytic viruses such as HSV-1, reovirus, vaccinia virus, and measles virus induce the production of numerous cytokines and increase the expression of co-stimulatory molecules (CD80 and CD86) and class II MHC [25-28]. Using HSV d120 HSVd120lacking the immediate early gene ICP 4 infected cell protein (ICP)4, Benencia et al. [2] showed that infected tumor cells induced DC maturation more efficiently than tumor cells killed by ultraviolet irradiation. Ma et al. [20] also reported that the supernatants of retinoblastoma cells improved the antigen-presenting function of DCs and their ability to activate T cells. However, the role of secreted molecules from HSV-1-infected SCC in DCs-mediated T cell activation had not been studied.

Previously, we prepared the supernatants of RH2-infected SCCVII cells in which virus infectivity was lost by ultraviolet irradiation and demonstrated the ability of the supernatants to enhance antitumor immunity in tumor-bearing mice [13]. In the present study, we examined the ability of supernatants collected $24 \mathrm{~h}$ after infection, i.e., Med 24,to induce the maturation of DCs prepared from the spleens of tumor-bearing mice. A significant increase in TNF- $\alpha$ occurred in the culture medium of DCs treated with Med24, indicating that Med 24 had the ability to induce DCs in order to produce cytokines. When we used DCs pulse-treated with Med24 or MedC, i.e., Med24-DCs and MedC-DCs, for cocultivation with $\mathrm{CD}^{+} \mathrm{T}$ cells or $\mathrm{CD} 8^{+} \mathrm{T}$ cells, an increase of INF- $\alpha$ production was observed as compared with unstimulated DCs (MEM-DC) It is possible that DCs as well as T cell produce IFN- $\gamma$ because DCs were included in the co-culture study. However, since the ratio of T cells and DCs was 9:1, the contribution of DCs on IFN- $\gamma$ production may be low. Therefore, it is considered that DCs contributed to successful presentation of MHC class I and MHC class II antigenic peptides on $\mathrm{CD}^{+}{ }^{+} \mathrm{T}$ cells and $\mathrm{CD} 8^{+} \mathrm{T}$ cells and the production of INF- $\gamma$ by these T cells.
In this regard, damage-associated molecular patterns (DAMPs) are molecules derived from normal cells that can initiate and perpetuate immunity in response to cell/tissue damage in the absence of pathogenic infection. They can be proteins, DNA, RNA, or metabolic products [4,29]. Protein DAMPs include intracellular proteins, such as high-mobility group box 1 (HMGB1), heat-shock proteins (HSPs), and proteins in the intracellular matrix that are generated following injury $[4,29,30]$. We also reported that DAMPs, ATP and HMGB1, were released extracellularly, while another DAMP, clareticulin, translocated to the cell membrane, indicating the production of DAMPs from SCC cells by on colyitc HSV-1[13]. Therefore, DMPAs in the supernatants of the RH2infected cells may contribute to the ability of Med24 to enhance tumor immunity [31].

In vivo studies, Workenhe et al [32] reported that tumors infected with the (ICP0)-deficient HSV-1 (dICP0) showed significant higher levels of DAMPs, with higher numbers of antigen-presenting cells within the tumor and increased antigenspecific CD8 ${ }^{+}$T-cell levels in the peripheral blood. Oncolytic HSV-1 $\mathrm{T}$ - vec injections into melanoma patients have also been reported to induce local and systemic antigen-specific $\mathrm{T}$ cell responses and decrease $\mathrm{CD}^{+}{ }^{+} \mathrm{FoxP}^{+}$regulatory $\mathrm{T}$ cells, $\mathrm{CD}^{+}{ }^{+}$FoxP3 ${ }^{+}$suppressor $\mathrm{T}$ cells, and myeloid-derived suppressive cells [33]. We also performed in vivo experiments to determine whether cytotoxic $\mathrm{T}$ cells can be activated following administration of Med24. Mice were treated three times with an intratumoral injection of Med24 or MedC. When splenocytes of the treated mice were co-cultured with SCCVII cells, more INF- $\gamma$ was produced in the Med24-treated group than in the MedC-treated group. Since the effects of Med24 on the production of IFN- $\gamma$ were not observed by a co-culture of splenocytes and Yac-1 cells, the enhanced immunity was found to be specifically directed to SCCVII cells. Splenocytes from Med24-treated mice were more cytotoxic to SCCVII cells than the splenocytes of MedC-treated mice. Using flow cytometry, we also found that a larger number of infiltrating CD8 ${ }^{+} \mathrm{T}$ cells were detected in the tumors of mice treated with Med24. This enhanced infiltration was observed on the non-injected side of the tumor, indicating the activation of systemic antitumor immunity. Together, we concluded that the intratumoral injection of molecules released from RH2-infected SCCVII cells promoted adaptive antitumor immunity through the activation of DCs, CD4 ${ }^{+}$ $\mathrm{T}$ cells, and cytotoxic $\mathrm{CD}^{+} \mathrm{T}$ cells.

\section{Conclusion}

We herein suggested that DCs in SCCVII tumor-bearing mice matured in the presence of the supernatants of RH2-infected SCCVII cells and activated CD $4^{+} \mathrm{T}$ cells and $\mathrm{CD} 8^{+} \mathrm{T}$ cells. This may promote the immunological effects of oncolytic HSV-1 RH2 on SCC cells. Therefore, the supernatants of the HSV-1-infected SCC cells can be the source to identify the unique molecules that elicit subsequent antitumor immunity. 


\section{References}

1. Thomas DL, Fraser NW. HSV-1 therapy of primary tumors reduces the number of metastases in an immune-competent model of metastatic breast cancer. Mol Ther. 2003;8(4):543-551.

2. Benencia F, Courrèges MC, Conejo-García JR, Mohammed-Hadley A, Coukos G. Direct vaccination with tumor cells killed with ICP4-deficient HSVd120 elicits effective antitumor immunity. Cancer Biol Ther. 2006;5(7):867-874

3. Bartlett DL, Liu Z, Sathaiah M, Ravindranathan R, Guo Z, He Y. Oncolytic viruses as therapeutic cancer vaccines. MolCancer. 2013; 12(1):103. doi 10.1186/1476-4598-12-103.

4. Guo ZS, Liu Z, Bartlett DL. Oncolytic immunotherapy: dying the right way is a key to eliciting potent antitumor immunity. Front Oncol. 2014;4:74. doi: 10.3389/fonc.2014.00074.

5. Yura Y, Hamada M. Development of oncolytic virothearpy for the treatment of oral cancer -At the waiting stage for clinical use. Oral Sci Int 2017;14(1):1-12. https://doi.org/10.1016/S1348-8643(16)30016-7.

6. Russell SJ, Peng KW, Bell JC. Oncolytic virotherapy. Nat Biotechnol. 2012;30(7):658-670. doi: 10.1038/nbt.2287.

7. Harrington KJ, Puzanov I, Hecht JR, Hodi FS, Szabo Z, Murugappan $\mathrm{S}$, et al. Clinical development of talimogenelaherparepvec (T-VEC): a modified herpes simplex virus type-1-derived oncolytic immunotherapy Expert Rev Anticancer Ther. 2015;15(12):1389-1403. doi: 10.1586/14737140.2015.1115725.

8. Nussenzweig MC, Steinman RM, Gutchinov B, Cohn ZA. Dendritic cells are accessory cells for the development of anti-trinitrophenyl cytotoxic T lymphocytes. J Exp Med. 1980;152(4):1070-1084.

9. Pierre P, Turley SJ, Gatti E, Hull M, Meltzer J, Mirza A, et al. Developmental regulation of MHC class II transport in mouse dendritic cells. Nature. 1997;388(6644):787-792. DOI:10.1038/42039.

10. Hengel H, Lindner M, Wagner H, Heeg K. Frequency of herpes simplex virusspecific murine cytotoxic T lymphocyte precursors in mitogen- and antigendriven primary in vitro T cell responses. J Immunol. 1987;139(12):41964202.

11. Rajasagi NK, Kassim SH, Kollias CM, Zhao X, Chervenak R, Jennings SR. CD4+ $\mathrm{T}$ cells are required for the priming of CD8+ T cells following infection with herpes simplex virus type 1.J Virol. 2009;83(10):5256-5268. doi: 10.1128/ JVI.01997-08.

12. Meshii N, Takahashi G, Okunaga S, Hamada M, Iwai S, Takasu A, et al. Enhancement of systemic tumor immunity for squamous cell carcinoma cells by an oncolytic herpes simplex virus. Cancer Gene Ther 2013;20(9):493-498. doi: 10.1038/cgt.2013.45.

13. Takasu A, Masui A, Hamada M, Imai T, Iwai S, Yura Y. Immunogenic cell death by oncolytic herpes simplex virus type 1 in squamous cell carcinoma cells.Cancer Gene Ther. 2016;23(4):107-113. doi: 10.1038/cgt.2016.8.

14. Takahashi G, Meshii N, Hamada M, Iwai S, Yura Y. Sequence of a fusogenic herpes simplex virus, RH2, for oncolytic virotherapy. J Gen Virol. 2013;94:726-737. doi: 10.1099/vir.0.044834-0.

15. Schlecht G, Mouriès J, Poitrasson-Rivière $M$, Leclerc C, Dadaglio G. Purification of splenic dendritic cells induces maturation and capacity to stimulate Th1 response in vivo. Int Immunol. 2006;18(3):445-452. doi:10.1093/intimm/dxh384.

16. Ing R, Segura M, Thawani N, Tam M, Stevenson MM. Interaction of mouse dendritic cells and malaria-infected erythrocytes: uptake, maturation, and antigen presentation. J Immunol. 2006;176(1):441-450.

17. Soroosh P, Ine S, Sugamura K, Ishii N. OX40-OX40 ligand interaction through $\mathrm{T}$ cell-T cell contact contributes to CD4 T cell longevity. J Immunol 2006;176(10):5975-5987.
18. Simma O, Zebedin E, Neugebauer N, Schellack C, Pilz A, Chang-Rodriguez $S$, et al. Identification of an indispensable role for tyrosine kinase 2 in CTL-mediated tumor surveillance. Cancer Res. 2009;69(1):203-211. doi: 10.1158/0008-5472.CAN-08-1705.

19. Li H, Liu L, Tao Y, Zhao P, Wang F, Huai L, et al. Effects of polysaccharides from Pholiotanameko on maturation of murine bone marrow-derived dendritic cells. Int J Biol Macromol. 2014;63:188-197. doi: 10.1016/j. ijbiomac.2013.11.002.

20. Ma J, Han H, Ma L, Liu C, Xue X, Ma P, et al. The immunostimulatory effects of retinoblastoma cell supernatant on dendritic cells. Protein Cell. 2014;5(4):307-316. doi: 10.1007/s13238-014-0029-0.

21. Kim Y, Clements DR, Sterea AM, Jang HW, Gujar SA, Lee PW. Dendritic cells in oncolytic virus-based anti-cancer therapy. Viruses. 2015;7(12):65066525. doi: 10.3390/v7122953.

22. Fleischer B, Schrezenmeier H, Wagner H. Function of the CD4 and CD8 molecules on human cytotoxic $\mathrm{T}$ lymphocytes: regulation of $\mathrm{T}$ cell triggering. J Immunol. 1986;136(5):1625-1628.

23. Bennett SR, Carbone FR, Karamalis F, Flavell RA, Miller JF, Heath WR. Help for cytotoxic-T-cell responses is mediated by CD40 signalling. Nature. 1998;393(6684):478-480. doi:10.1038/30996.

24. Gujar SA, Lee PW. Oncolytic virus-mediated reversal of tumor antigen presentation. Front Oncol. 2014;4:77. doi: 10.3389/fonc.2014.00077.

25. Greiner S, Humrich JY, Thuman P, Sauter B, Schuler G, Jenne L. The highly attenuated vaccinia virus strain modified virus Ankara induces apoptosis in melanoma cells and allows bystander dendritic cells to generate a potent anti-tumoral immunity. Clin Exp Immunol. 2006;146(2):344-353.

26. Benencia F, Courrèges MC, Fraser NW, Coukos G. Herpes virus oncolytic therapy reverses tumor immune dysfunction and facilitates tumor antigen presentation. Cancer Biol Ther. 2008;7(8):1194-1205.

27. Gujar SA, Marcato P, Pan D, Lee PW. Reovirusvirotherapy overrides tumor antigen presentation evasion and promotes protective antitumor immunity. Mol Cancer Ther. 2010;9(11):2924-2933. doi: 10.1158/15357163.

28. Guillerme JB, Boisgerault N, Roulois D, Ménager J, Combredet C, Tangy $\mathrm{F}$, et al. Measles virus vaccine-infected tumor cells induce tumor antigen cross-presentation by human plasmacytoid dendritic cells. Clin Cancer Res. 2013;19(5):1147-1158. doi: 10.1158/1078-0432.

29. Kono H, Rock KL. How dying cells alert the immune system to danger. Nat Rev Immunol. 2008;8(4):279-289. doi: 10.1038/nri2215.

30. Klune JR, Dhupar R, Cardinal J, Billiar TR, Tsung A. HMGB1: endogenous danger signaling. Mol Med. 2008;14(7-8):476-484. doi: 10.2119/200800034.

31. Tada S, Hamada M, Yura Y. Proteomic analysis of secretomes of oncolytic herpes simplex virus-infectedsquamous cell carcinoma cells. Cancers (Basel). 2018;10(2). doi: 10.3390/cancers10020028.

32. Workenhe ST, Simmons G, Pol JG, Lichty BD, Halford WP, Mossman KL. Immunogenic HSV-mediated on colysis shapes the antitumor immune response and contributes to therapeutic efficacy. Mol Ther. 2014;22(1):123131. doi: 10.1038/mt.2013.238.

33. Kaufman HL, Kim DW, DeRaffele G, Mitcham J, Coffin RS, Kim-Schulze S. Local and distant immunity induced by intralesional vaccination with an oncolytic herpes virus encoding GM-CSF in patients with stage IIIc and IV melanoma. Ann Surg Oncol. 2010: 17(3):718-730. doi: 10.1245/s10434009-0809-6. 